Supporting Information

\title{
Probing Lipophilic Adamantyl Group as the P1-Ligand for HIV-1 Protease Inhibitors: Design, Synthesis, Protein X-ray Structural Studies, and Biological Evaluation
}

\author{
Arun K. Ghosh, Heather L. Osswald, Kristof Glauninger, Johnson Agniswamy, Yuan-Fang
} Wang, Hironori Hayashi, Manabu Aoki, Irene T. Weber, and Hiroaki Mitsuya

Table of Contents

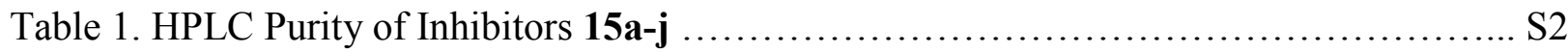

Table 2. High Resolution Mass Spectrometry Data for Inhibitors 15a-j ................... S3

Table 3. Crystallographic data collection and refinement statistics ...................... S4

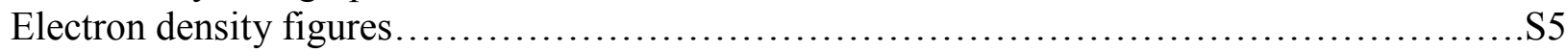


Table 1. Analytical Purity of Inhibitors 15a-j determined by HPLC.

\begin{tabular}{|c|c|c|c|c|}
\hline Inhibitor & $\begin{array}{c}\text { Retention } \\
\text { Time } \\
\text { (min) }\end{array}$ & Column & $\begin{array}{c}\text { Flow Rate } \\
\text { (ml/min) }\end{array}$ & $\begin{array}{c}\text { Purity } \\
(\%)\end{array}$ \\
\hline $15 a$ & 22.90 & $\begin{array}{c}\text { YMC Pack ODS-A } \\
5 \mu \mathrm{M} 250 \times 4.6 \mathrm{~mm} \text { ID }\end{array}$ & 1 & 97.3 \\
\hline $15 b$ & 22.85 & $\begin{array}{c}\text { YMC Pack ODS-A } \\
5 \mu \mathrm{M} 250 \times 4.6 \mathrm{~mm} \text { ID }\end{array}$ & 1 & 96.8 \\
\hline $15 c$ & 21.19 & $\begin{array}{c}\text { YMC Pack ODS-A } \\
5 \mu \mathrm{M} 250 \times 4.6 \mathrm{~mm} \text { ID }\end{array}$ & 1 & 98.3 \\
\hline 15d & 22.75 & $\begin{array}{c}\text { YMC Pack ODS-A } \\
5 \mu \mathrm{M} 250 \times 4.6 \mathrm{~mm} \text { ID }\end{array}$ & 1 & 95.3 \\
\hline $15 e$ & 22.76 & $\begin{array}{c}\text { YMC Pack ODS-A } \\
5 \mu \mathrm{M} 250 \times 4.6 \mathrm{~mm} \text { ID }\end{array}$ & 1 & 96.1 \\
\hline $15 f$ & 20.95 & $\begin{array}{c}\text { YMC Pack ODS-A } \\
5 \mu \mathrm{M} 250 \times 4.6 \mathrm{~mm} \text { ID }\end{array}$ & 1 & 95.4 \\
\hline $15 \mathrm{~g}$ & 22.25 & $\begin{array}{c}\text { YMC Pack ODS-A } \\
5 \mu \mathrm{M} 250 \times 4.6 \mathrm{~mm} \text { ID }\end{array}$ & 1 & 97.4 \\
\hline $15 \mathrm{~h}$ & 22.97 & $\begin{array}{c}\text { YMC Pack ODS-A } \\
5 \mu \mathrm{M} 250 \times 4.6 \mathrm{~mm} \text { ID }\end{array}$ & 1 & 96.5 \\
\hline $15 i$ & 23.65 & $\begin{array}{c}\text { YMC Pack ODS-A } \\
5 \mu \mathrm{M} 250 \times 4.6 \mathrm{~mm} \text { ID }\end{array}$ & 1 & 97.1 \\
\hline $15 j$ & 23.23 & $\begin{array}{c}\text { YMC Pack ODS-A } \\
5 \mu \mathrm{M} 250 \times 4.6 \mathrm{~mm} \text { ID }\end{array}$ & 1 & 98.4 \\
\hline
\end{tabular}

HPLC Conditions: $\mathrm{t}=0-2 \min \left[0: 100 \mathrm{MeCN}: \mathrm{H}_{2} \mathrm{O}\right], \mathrm{t}=2-22 \min$ [gradient 0:100 $\mathrm{MeCN}: \mathrm{H}_{2} \mathrm{O}$ to 100:0 MeCN: $\mathrm{H}_{2} \mathrm{O}$ ], 22-25 $\min$ [100:0 MeCN: $\left.\mathrm{H}_{2} \mathrm{O}\right] ; 254 \mathrm{nM}$ detection; $\mathrm{T}=30^{\circ} \mathrm{C}$ 
Table 2. High Resolution Mass Spectrometry Data for Inhibitors 15a-j

\begin{tabular}{|l|l|l|l|l|l|}
\hline Inhibitor & Molecular & HRMS & Ion & Calculated & Found \\
& Formula & Technique & & & \\
\hline $\mathbf{1 5 a}$ & $\mathrm{C}_{30} \mathrm{H}_{46} \mathrm{~N}_{2} \mathrm{O}_{7} \mathrm{~S}$ & ESI & {$[M+\mathrm{H}]^{+}$} & 579.3104 & 579.31235 \\
\hline $\mathbf{1 5 b}$ & $\mathrm{C}_{30} \mathrm{H}_{46} \mathrm{~N}_{2} \mathrm{O}_{7} \mathrm{~S}$ & ESI & {$[M+\mathrm{H}]^{+}$} & 579.3104 & 579.31175 \\
\hline $\mathbf{1 5 c}$ & $\mathrm{C}_{30} \mathrm{H}_{46} \mathrm{~N}_{2} \mathrm{O}_{7} \mathrm{~S}$ & ESI & {$[M+\mathrm{H}]^{+}$} & 579.3104 & 579.31235 \\
\hline $\mathbf{1 5 d}$ & $\mathrm{C}_{32} \mathrm{H}_{48} \mathrm{~N}_{2} \mathrm{O}_{8} \mathrm{~S}$ & ESI & {$[M+\mathrm{H}]^{+}$} & 621.3210 & 621.32166 \\
\hline $\mathbf{1 5 e}$ & $\mathrm{C}_{32} \mathrm{H}_{48} \mathrm{~N}_{2} \mathrm{O}_{8} \mathrm{~S}$ & ESI & {$[M+\mathrm{H}]^{+}$} & 621.3210 & 621.32200 \\
\hline $\mathbf{1 5 f}$ & $\mathrm{C}_{31} \mathrm{H}_{47} \mathrm{~N}_{3} \mathrm{O}_{7} \mathrm{~S}$ & ESI & {$[M+\mathrm{H}]^{+}$} & 606.3213 & 606.32100 \\
\hline $\mathbf{1 5 g}$ & $\mathrm{C}_{34} \mathrm{H}_{44} \mathrm{~N}_{2} \mathrm{O}_{8} \mathrm{~S}$ & ESI & {$[M+\mathrm{H}]^{+}$} & 641.2897 & 641.29091 \\
\hline $\mathbf{1 5 h}$ & $\mathrm{C}_{35} \mathrm{H}_{46} \mathrm{~N}_{2} \mathrm{O}_{8} \mathrm{~S}$ & ESI & {$[M+\mathrm{H}]^{+}$} & 655.3054 & 655.30729 \\
\hline $\mathbf{1 5 i}$ & $\mathrm{C}_{33} \mathrm{H}_{50} \mathrm{~N}_{2} \mathrm{O}_{8} \mathrm{~S}$ & ESI & {$[M+\mathrm{H}]^{+}$} & 635.3367 & 635.33882 \\
\hline $\mathbf{1 5 j}$ & $\mathrm{C}_{33} \mathrm{H}_{50} \mathrm{~N}_{2} \mathrm{O}_{8} \mathrm{~S}$ & ESI & {$[M+\mathrm{H}]^{+}$} & 635.3367 & 635.33875 \\
\hline
\end{tabular}


Table 3. Crystallographic data collection and refinement statistics

\begin{tabular}{|c|c|c|c|}
\hline Complex Name & $\begin{array}{l}\mathrm{PR}_{\mathrm{WT}} / \\
\text { inhibitor 15d }\end{array}$ & $\begin{array}{l}\mathrm{PR}_{\mathrm{WT}} / \\
\text { inhibitor } \mathbf{1 5 h}\end{array}$ & $\begin{array}{l}\mathrm{PR}_{\mathrm{WT}} / \\
\text { inhibitor } \mathbf{1 5 i}\end{array}$ \\
\hline PDB Code & $5 \mathrm{JFP}$ & $5 \mathrm{JFU}$ & 5JG1 \\
\hline Space group & $\mathrm{P} 2{ }_{1} 2{ }_{1} 2$ & $\mathrm{P} 2{ }_{1} 2_{1} 2$ & $\mathrm{P} 2{ }_{1} 2{ }_{1} 2$ \\
\hline \multicolumn{4}{|l|}{ Unit cell dimensions: $(\AA)$} \\
\hline $\mathrm{a}$ & 58.10 & 58.96 & 58.04 \\
\hline $\mathrm{b}$ & 86.14 & 86.38 & 86.41 \\
\hline $\mathrm{c}$ & 45.95 & 45.92 & 45.99 \\
\hline Resolution range (final shell) $(\AA)$ & $\begin{array}{l}50-1.49 \\
(1.54-1.49)\end{array}$ & \begin{tabular}{|l|}
$50-1.70$ \\
$(1.76-1.70)$
\end{tabular} & $\begin{array}{l}50-1.16 \\
(1.20-1.16)\end{array}$ \\
\hline Unique reflections & 36,868 & 26,464 & 77,803 \\
\hline Wilson B-factor & 14.6 & 19.2 & 9.8 \\
\hline $\mathrm{R}_{\text {merge }}(\%)$ overall (final shell) & $9.7(49.2)$ & $7.6(48.7)$ & $9.8(44.4)$ \\
\hline $\mathrm{I} / \sigma(\mathrm{I})$ overall (final shell) & $11.8(4.5)$ & $19(3.0)$ & $19.2(2.0)$ \\
\hline $\begin{array}{l}\text { Completeness (\%) overall (final } \\
\text { shell) }\end{array}$ & $97.5(96.1)$ & $99.9(99.7)$ & $94.9(64.3)$ \\
\hline Redundancy (final shell) & $4.8(4.4)$ & $5.9(4.8)$ & $7.7(2.2)$ \\
\hline $\mathrm{R}(\%)$ & 21.5 & 22.8 & 15.8 \\
\hline $\mathrm{R}_{\text {free }}(\%)$ & 23.7 & 27.8 & 19.2 \\
\hline $\begin{array}{l}\text { No. of solvent atoms } \\
\text { (total occupancies) }\end{array}$ & $146(134.6)$ & $96(91)$ & $194(167.4)$ \\
\hline \multicolumn{4}{|l|}{ RMS deviation from ideality } \\
\hline Bonds $(\AA)$ & 0.009 & 0.006 & 0.014 \\
\hline Angle distance $(\AA)$ & 0.026 & 0.023 & 0.030 \\
\hline \multicolumn{4}{|l|}{ Average B-factors $\left(\AA^{2}\right)$} \\
\hline Main-chain atoms & 13.9 & 18.6 & 11.6 \\
\hline Side-chain atoms & 20.3 & 25.6 & 17.6 \\
\hline Whole chain atoms & 17.0 & 21.9 & 14.5 \\
\hline Inhibitor & 17.8 & 22.7 & 13.5 \\
\hline Solvent & 25.1 & 26.3 & 23.9 \\
\hline $\begin{array}{l}\text { RMS deviation to DRV (2IEN) } \\
(\AA)\end{array}$ & 0.33 & 0.29 & 0.35 \\
\hline
\end{tabular}



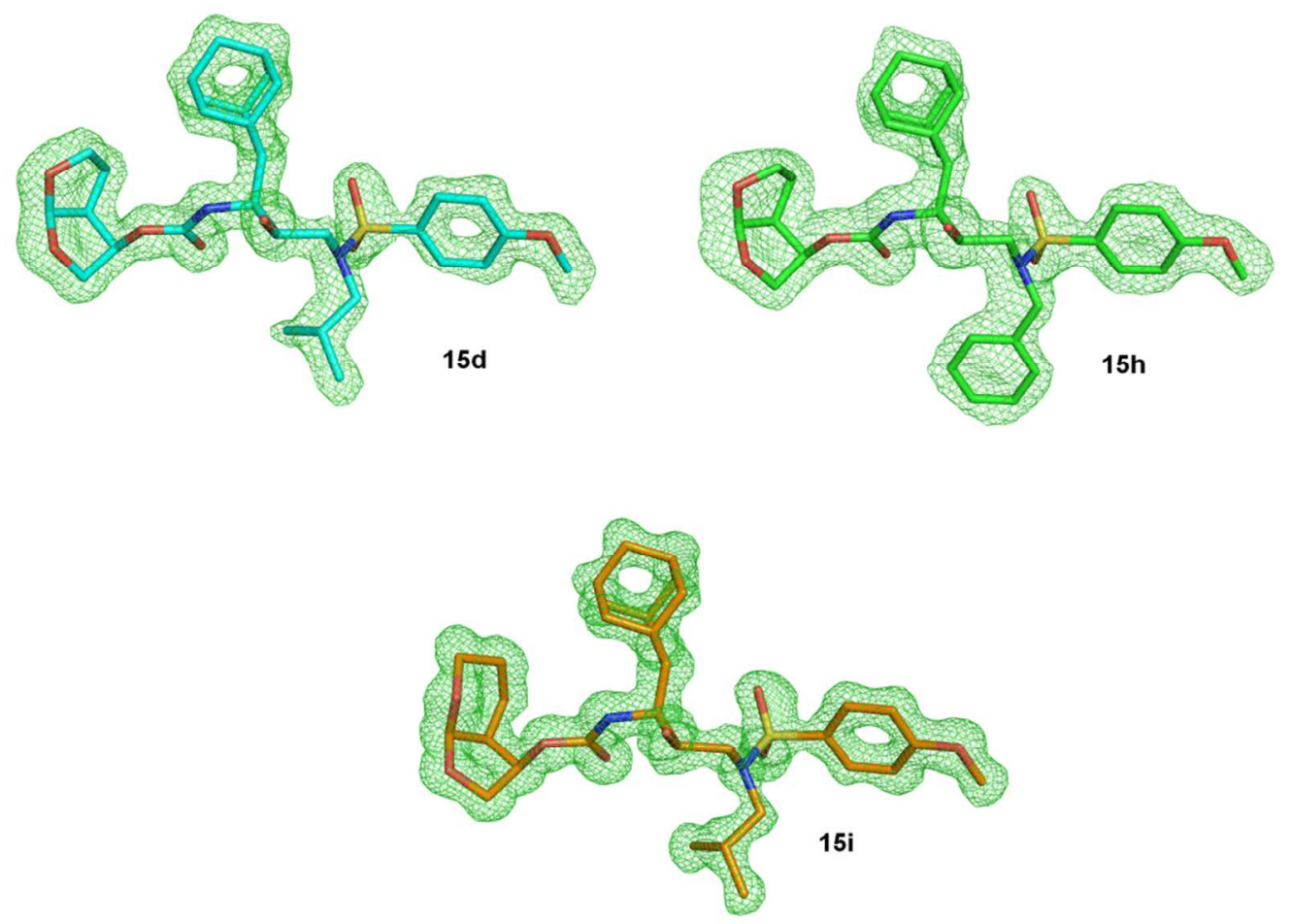

$\mathrm{F}_{\mathrm{o}}-\mathrm{F}_{\mathrm{c}}$ omit map of inhibitors $\mathbf{1 5 d}$ (turquoise), 15h (green) and 15i (Yellow) contoured at $3 \sigma$ level. 